\title{
Prevalencia de signos y síntomas de trastornos temporomandibulares previo a tratamiento de ortodoncia en una población de Santiago, Chile.
}

\author{
Prevalence of signs and symptoms of temporomandibular \\ disorders before orthodontic treatment in a population of \\ Santiago, Chile.
}

\author{
Claudia Larenas-Calderón ${ }^{1 *}$, Loreto Saavedra-Layera ${ }^{2}$, Cristian Vergara-Núñez ${ }^{3}$, Natalia Spano-Perez ${ }^{4}$.
}

1. Cirujano dentista, CESFAM Bellavista de la Florida, Santiago, Chile.

2. Cirujano dentista, CESFAM Padre Vicente Irarrázabal, Santiago, Chile.

3. Cirujano dentista, Departamento del Niño y Ortopedia Dentomaxilar, Universidad de Chile, Santiago, Chile.

4. Fonoaudióloga, Centro Infantil Amanaki, Santiago, Chile.

* Correspondencia autor. Claudia Larenas Calderón | Alonso de Camargo interior 6221, Las Condes, Santiago, Chile. | Teléfono: +56 976482640 |

E-mail: cclarenasc@gmail.com

Trabajo recibido el 18/04/2018.

Aprobado para su publicación el 15/09/2018

\section{RESUMEN}

Objetivo: El objetivo de este estudio fue establecer la prevalencia de signos y síntomas de trastornos temporomandibulares (TTM) en pacientes con maloclusión. Materiales y métodos: Estudio transversal descriptivo. Se revisaron de forma manual 951 fichas clínicas de pacientes ingresados en el Postítulo de Ortodoncia de la Facultad de Odontología de Universidad de Chile (FOUCH) entre los años 2013 y 2015 . Se utilizaron los programas Excel 2013, Stata v. 12 y para la comparación entre grupos etarios se utilizó la prueba estadística de Kruskall Wallis. Resultados: Del total de pacientes con maloclusión, el $43,9 \%$ presentaron al menos un signo o síntoma de TTM, siendo la desviación mandibular el signo más prevalente con un $43,2 \%$, seguido por el ruido articular con un $12,6 \%$. El dolor fue el síntoma menos prevalente con un $2 \%$. Los adultos fueron los que presentaron mayor prevalencia de signos y síntomas de TTM. No hubo diferencia estadística entre géneros. Conclusión: Debido a que los signos y síntomas de TTM son una condición tan común en pacientes con maloclusión, es necesaria la evaluación de la ATM siempre que se piense en iniciar un tratamiento ortodóncico, para así establecer un adecuado plan de tratamiento.

PALABRAS CLAVE:

Articulación temporomandibular; Trastornos temporomandibulares; Maloclusión.

Rev. Clin. Periodoncia Implantol. Rehabil. Oral Vol. 11(3); 160-163, 2018.

\begin{abstract}
Objective: The aim of this study was to establish the prevalence of signs and symptoms of temporomandibular disorders (TMD) in patients with malocclusion. Materials and methods: Cross-sectional descriptive study. A total of 951 clinical records were reviewed, of patients admitted in the Postgraduate Program of Orthodontia from the Faculty of Dentistry of the University of Chile, between the years 2013 and 2015. The programs Excel 2013 and Stata v. 12 were used, and for the comparison between the age groups the statistical test of Kruskall Wallis was used. Results: Of the total number of patients with malocclusion, $43.85 \%$ had at least one sign or symptom of TMD, being the mandibular deviation the most prevalent sign with $43.22 \%$, followed by joint noise with $12.62 \%$. Pain was the least prevalent symptom with $2 \%$. The adults group had the most prevalence of signs and symptoms of TMD. There was no statistical difference between genders. Conclusion: Since TMD signs and symptoms are such a common condition in patients with malocclusion, the assessment of TMJ is necessary to consider when planning an orthodontic treatment, to establish an adequate treatment plan. .
\end{abstract}

KEY WORDS:

Temporomandibular joint; Temporomandibular disorders; Malocclusion.

Rev. Clin. Periodoncia Implantol. Rehabil. Oral Vol. 11(3); 160-163, 2018.

\section{INTRODUCCIÓN}

Los trastornos témporomandibulares (TTM) se pueden definir como un grupo de condiciones músculo-esqueletales que afectan la articulación témporomandibular (ATM), los músculos masticadores y estructuras asociadas, generando un conjunto de signos y síntomas, siendo los más relevantes: dolor articular, ruidos articulares, alteraciones de la dinámica mandibular y dolor miofascial. Entre este conjunto de signos y síntomas, el dolor aparece como la principal causa de consultas y solicitudes de tratamiento ${ }^{(1)}$.

Por otro lado, las maloclusiones, según la Organización Mundial de la Salud (OMS) es una entidad patológica que ocupa el tercer lugar entre 
las enfermedades bucales ${ }^{(2)}$, por lo que se clasifica como un problema de salud pública bucal. Ésta no solo afecta a nivel funcional y estético, sino que también influye en aspectos psicosociales y en la calidad de vida de las personas, especialmente de adolescentes ${ }^{(3)}$.

Numerosas teorías etiológicas y terapéuticas sobre TTM se basan en una presunta correlación entre éstos y las maloclusiones ${ }^{(4,5)}$. Estudios internacionales recientes estiman una cifra promedio de $27 \%$ en la prevalencia de TTM en pacientes con maloclusión ${ }^{(6,7,8)}$. Sin embargo, la mayoría de los estudios no han podido justificar los tratamientos ortodóncicos como una alternativa para prevenir o tratar los TTM.

Sin embargo, a pesar de aquello, no parece razonable y no podría justificarse desde un punto de vista técnico o incluso legal, comenzar un tratamiento de ortodoncia en un paciente portador de TTM sin una evaluación previa de un especialista en disfunción.

Debido a que los TTM son una condición común en pacientes con maloclusión, es necesaria la evaluación de las ATMs siempre que se piense en iniciar un tratamiento ortodóncico, para poder establecer un adecuado plan de tratamiento que permita la corrección de ambos y así minimizar el tiempo de intervención y alcanzar resultados favorables en la estética facial, la función articular y los músculos masticatorios.

El presente estudio tiene como objetivo establecer la prevalencia de signos y síntomas de TTM en pacientes con maloclusión con el fin de poder aportar información relevante sobre la presentación de este trastorno en pacientes con esta condición.

\section{MATERIALES Y MÉTODOS}

Se realizó un estudio transversal descriptivo. Se revisaron de forma manual las fichas clínicas de los pacientes que ingresaron al Postítulo de Ortodoncia de la Facultad de Odontología de la Universidad de Chile (FOUCH) entre los años 2013 y 2015 (1.216 fichas clínicas). La muestra se determinó estimando un promedio de prevalencia de signos y síntomas de TTM en pacientes con maloclusión, mayor al $50 \%$, basado en estudios internacionales ${ }^{(7,8,9)}$. Además, la fórmula se basó en el número de personas con maloclusión en Chile $(\mathrm{N})$, estimando que un $70 \%$ de la población chilena, según el CENSO 2012 (INE), presenta esta condición ${ }^{(10)}$. Se realizó una selección de acuerdo a criterios de inclusión y de exclusión, hasta obtener una muestra de 951 fichas clínicas.

\section{Criterios de inclusión}

Se consideraron fichas clínicas que se encontraban completas en las cuales los pacientes reunían las siguientes características: ingresados entre los años 2013 y 2015, diagnosticados con maloclusión, presencia de dentición mixta y/o permanente y sin historia previa de ortodoncia.

\section{Criterios de exclusión}

Se excluyeron aquellas fichas donde los pacientes reunían las siguientes características: ingresado año anterior al 2013, presencia de dentición temporal, presencia de factores de riesgo de TTM: bruxismo, trauma en región maxilomandibular y enfermedades sistémicas que pudieran afectar el funcionamiento articular (artritis, artrosis, osteoporosis, reumatismo, lupus, etc). Presencia de algún síndrome con afección en ATM (Síndrome de Goldenhar, microsomía hemifacial), uso previo de aparatos ortodóncicos.

La información recolectada fue transferida a planillas Excel. Cabe destacar que no se consideraron los datos personales sensibles de los pacientes.

Las variables independientes correspondieron a: género (femenino o masculino), grupo etario (según clasificación de la OMS: escolar, adolescente, adulto joven, adultez). Las variables dependientes correspondieron a: desviación a la derecha, desviación a la izquierda, ruido articular, salto articular, dolor, presencia de al menos un signo o síntoma de TTM, cantidad de signos y/o síntomas de TTM.

\section{Análisis estadístico}

Los datos obtenidos fueron tabulados y procesados en los programas de computación MS-Excel 2013 y Stata v. 12. Para los cálculos de prevalencias de signos y síntomas de TTM se utilizaron porcentajes, para la comparación de las variables por grupos etarios (cuatro grupos: escolar, adolescente, adulto joven, adultez) se utilizó la prueba estadística de Kruskall Wallis. Se consideró que hubo diferencia estadística cuando $\mathrm{p} \leq 0,05$.

\section{RESULTADOS}

Del total de fichas clínicas de pacientes seleccionadas, el rango de edad osciló entre los 6 y los 60 años, con un promedio de 16,9 $\pm 7,5$ años. Del total de la muestra, $57,1 \%$ correspondieron a individuos del género femenino ( $n=543)$, mientras que $42,9 \%$ fueron del sexo masculino $(n=408)$. El mayor número de individuos estuvo en el rango de 10 a 19 años (adolescentes) ( $n=594)$ y el menor número de individuos estuvo en el rango de 41 a 60 años (adultez) $(n=13)$.

\section{- Prevalencia de signos y síntomas de TTM}

La prevalencia de sujetos con al menos un signo o síntoma de TTM fue de $43,8 \%(n=417)$. El signo de TTM más frecuentemente encontrado fue el de desviación mandibular con un $43,2 \% \quad(n=411$, seguido por el ruido articular con un $12,6 \%(n=120)$. Por el contrario, el de menor prevalencia fue el dolor con un $2,0 \%(n=19)$. Además, se observa una mayor presencia de signos y/o síntomas de TTM en apertura, tal como se muestra en la tabla 1.

Tabla 1. Prevalencia de signos/síntomas de TTM en apertura y cierre en pacientes ingresados en el Postítulo de Ortodoncia entre los años 2013 y 2015

\begin{tabular}{llcll}
\hline Signo o síntoma de TTM & \multicolumn{3}{c}{ En apertura } & \multicolumn{2}{c}{ En cierre } \\
\hline & $\mathrm{n}$ & $\%$ & $\mathrm{~N}$ & $\%$ \\
Desviación a la derecha & 145 & 15,2 & 73 & 7,7 \\
Desviación a la izquierda & 154 & 16,2 & 96 & 10,1 \\
Ruido articular & 106 & 11,2 & 64 & 6,7 \\
Salto articular & 54 & 5,7 & 36 & 3,8 \\
Dolor & 15 & 1,6 & 9 & 0,9 \\
Total & 474 & 49,8 & 278 & 29,2 \\
\hline
\end{tabular}

Respecto a la cantidad de signos y/o síntomas presentados en los pacientes, el 19,8\% ( $n=189)$ presentó sólo un signo o síntoma de TTM, seguido por 2 signos y/o síntomas de TTM con un 17,2\% ( $n=164)$.

\section{- Prevalencia de signos y síntomas de TTM según grupos etarios}

Se encontró diferencia estadística entre los distintos grupos etarios respecto a la frecuencia de signos y síntomas de TTM $(p \leq 0,05)$, sin embargo, se debe tener en cuenta que la muestra de pacientes en adultez puede no ser significativa estadísticamente, pero sí mostrar una tendencia. Los adultos presentaron la mayor prevalencia de signos y/o síntomas de TTM con un $61,5 \%(n=8)$, seguido por los adultos jóvenes con un $54,9 \%(n=139)$. Por el contrario, los con menor prevalencia de signos y/o síntomas de TTM fueron los escolares con $24,1 \% \quad(n=22)$. Se encontró diferencias $(p \leq 0,05)$ entre los distintos grupos etarios con respecto a la presencia de ruido articular, no obstante, no hubo diferencias estadísticas con los otros signos y síntomas de TTM $(p>0,05)$. El signo más prevalente en escolar, adolescente y adulto joven fue el de desviación mandibular, mientras que en adultez fue tanto este signo como el ruido articular (tabla 2).

Tabla 2. Prevalencia de signos/síntomas de TTM de pacientes ingresados en el Postítulo de Ortodoncia entre los años 2013 y 2015, de acuerdo a cada grupo etario.

\begin{tabular}{llllllllllllll}
\hline & $\begin{array}{l}\text { Desviación a } \\
\text { Grupo Etario }\end{array}$ & $\begin{array}{l}\text { la derecha } \\
\text { la izquierda }\end{array}$ & \multicolumn{2}{l}{$\begin{array}{l}\text { Ruido } \\
\text { articular }\end{array}$} & \multicolumn{2}{l}{$\begin{array}{l}\text { Salto } \\
\text { articular }\end{array}$} & \multicolumn{2}{l}{ Dolor } \\
\hline Escolar & $\mathrm{n}$ & $\%$ & $\mathrm{n}$ & $\%$ & $\mathrm{n}$ & $\%$ & $\mathrm{n}$ & $\%$ & $\mathrm{n}$ & $\%$ \\
Adolescente & 11 & 117 & 19,1 & 11 & 12,1 & 0 & 0 & 6 & 6,6 & 1 & 1,1 \\
$\begin{array}{l}\text { Adulto } \\
\text { Joven }\end{array}$ & 60 & 23,7 & 66 & 23,1 & 56 & 22,1 & 20 & 7,9 & 4 & 1,6 \\
Adultez & 4 & 30,8 & 3 & 23,1 & 4 & 30,8 & 2 & 15,4 & 0 & 0 \\
TOTAL & 192 & 20,2 & 219 & 23,0 & 119 & 12,5 & 65 & 6,8 & 19 & 2,0 \\
\hline
\end{tabular}

\section{- Prevalencia de signos y síntomas de TTM según sexo}

La prevalencia de signos y/o síntomas de TTM según sexo, fue de un $44,9 \%$ para las mujeres $(n=244)$ y de un $42,4 \%$ para los hombres $(n=173)$. No se observaron diferencias estadísticas por sexo en la muestra estudiada $(p>0,05)$. El signo de TTM más prevalente tanto en hombres como mujeres fue el de desviación mandibular. Por el contrario, el de menor prevalencia fue el dolor (Tabla 3). No hubo diferencias estadísticas en la prevalencia de los distintos signos y síntomas de TTM entre ambos sexos $(p>0,05)$. 
Tabla 3. Prevalencia de signos/síntomas de TTM de pacientes ingresados en el Postítulo de Ortodoncia entre los años 2013 y 2015, según sexo.

\begin{tabular}{llllllllllll}
\hline Sexo & $\begin{array}{l}\text { Desviación a } \\
\text { la derecha }\end{array}$ & $\begin{array}{l}\text { Desviación a } \\
\text { la zquierda }\end{array}$ & $\begin{array}{l}\text { Ruido } \\
\text { articular }\end{array}$ & \multicolumn{3}{l}{$\begin{array}{l}\text { Salto } \\
\text { articular }\end{array}$} & Dolor \\
\hline & $n$ & $\%$ & $n$ & $\%$ & $n$ & $\%$ & $n$ & $\%$ & $n$ & $\%$ \\
Femenino & 119 & 21,9 & 128 & 23,6 & 70 & 12,9 & 40 & 7,4 & 9 & 1,7 \\
Masculino & 73 & 17,9 & 91 & 22,3 & 49 & 12,0 & 25 & 6,1 & 10 & 2,5 \\
TOTAL & 192 & 20,2 & 219 & 23,0 & 119 & 12,5 & 65 & 6,8 & 19 & 2,0 \\
\hline
\end{tabular}

\section{DISCUSIÓN}

Numerosos estudios epidemiológicos han examinado la prevalencia de TTM en poblaciones específicas ${ }^{(11,12,13,14)}$. En ellos los pacientes fueron interrogados respecto de sus síntomas y luego examinados en busca de signos clínicos asociados a los TTM. Estos tienen en común la búsqueda de prevalencia de signos y síntomas con la ausencia de diagnósticos específicos y con diferencias en sus metodologías y grupos de estudio. En promedio los trabajos antes citados muestran una prevalencia entre un 40 a $60 \%$ de la población estudiada (niños, adolescentes y adultos) con al menos un signo o síntoma de TTM. Estas variaciones en la prevalencia de TTM se deben, más que a las reales diferencias en las muestras de estudio, probablemente a: la falta de métodos de examen estandarizados válidos y confiables que hagan posible la comparación entre los diferentes estudios, a deficiencias metodológicas y a la falta de definición y caracterización de los TTM(15, 16).

En este estudio se obtuvo que un $43,9 \%$ de los pacientes presentó algún signo o síntoma de TTM, siendo los más prevalentes la desviación mandibular, seguido por el ruido articular. El síntoma menos frecuente fue el dolor. Además, se observó una mayor presencia de signos y/o síntomas de TTM en apertura. De acuerdo a la literatura disponible no existen trabajos similares a nivel nacional, ya sea por diferencia en las edades de los pacientes, distintos objetivos de los estudios y diferencias en las características de los pacientes. No hay estudios de prevalencia de signos y síntomas de TTM en pacientes con maloclusión. En un trabajo realizado por Díaz y cols. ${ }^{(17)}$, con el fin de determinar la prevalencia de TTM y las características asociadas a esta patología en una población chilena, se estudiaron 269 pacientes entre 18 y 80 años, obteniéndose una prevalencia de TTM del $53,5 \%$. Casassus ${ }^{(18)}$ reportó que de una muestra de 374 escolares, en Santiago, entre 10 y 14 años, el 12,3\% presentaba algún diagnóstico de TTM. En el 2005, Corsini y cols. ${ }^{(19)}$ determinaron que de una muestra de 116 pacientes entre 13 y 18 años, en Temuco, el $77,6 \%$ mostró uno o más signos y/o síntomas de TTM, siendo el ruido el más prevalente.

A nivel internacional, los estudios de prevalencia de signos y síntomas de TTM en pacientes con maloclusión, muestran un promedio mayor al $50 \%$. Sin embargo, según la literatura disponible, sólo se han realizado estudios similares, en pacientes con maloclusión, circunscritos a grupos etarios de adolescentes ${ }^{(7,8,9,20)}$. Los resultados del presente estudio son menores a los encontrados en la literatura ${ }^{(7,9)}$. En un estudio realizado por López y cols. ${ }^{(7)}$, donde se evaluó clínicamente la presencia de TTM, antes del tratamiento ortodóncico, demostró que de una muestra de 67 pacientes entre 9 y 18 años, el $100 \%$ presentó al menos un signo o síntoma de TTM, siendo el dolor muscular el síntoma más prevalente con un $79,1 \%$, seguido de la desviación mandibular con un $53,7 \%$. Asimismo Soto y cols. ${ }^{(9)}$ reportaron que de una muestra de 84 pacientes entre 12 y 19 años, el 88,1\% presentaba algún signo y/o síntoma de TTM. Estos resultados difieren con los del presente trabajo, donde la prevalencia en el grupo etario de adolescentes fue del $41,8 \%$, siendo el signo más prevalente la desviación mandibular con un $43,1 \%$. Por el contrario, la prevalencia de signos y/o síntomas de TTM en adolescentes es mayor a los reportados por Spalj y cols. ${ }^{(8)}$, donde se evaluaron 1.597 pacientes con maloclusión entre 11 y 19 años, sin historia previa de ortodoncia, obteniéndose un $22 \%$ de prevalencia de al menos un signo o síntoma de TTM. Estas diferencias podrían deberse a los distintos criterios de inclusión y exclusión utilizados, y a las diferencias metodológicas aplicadas.
Según López y cols.(7), el $62 \%$ de los pacientes mostraron una disfunción moderada, considerándose como tal la presencia de tres o cuatro signos y/o síntomas, excepto disminución de la máxima apertura. Estos resultados difieren con los del presente trabajo, donde se reportó que la cantidad más prevalentes eran 1 ó 2 signos y/o síntomas de TTM, siendo $19,8 \%$ y $17,2 \%$ respectivamente, no obstante, cabe mencionar que en este trabajo no se consideró la máxima apertura.

Al igual que en otros estudios se determinó que la prevalencia de signos y síntomas de TTM se incrementa con la edad ${ }^{(21,22)}$. En este estudio los adultos presentaron la mayor prevalencia, seguido por los adultos jóvenes. Por el contrario, los con menor prevalencia de signos y/o síntomas de TTM fueron los escolares. Existió diferencia estadística entre los distintos grupos etarios respecto a la frecuencia de signos y síntomas de TTM $(p \leq 0,05)$, sin embargo, se debe tener en cuenta que la muestra de pacientes en adultez puede no ser significativa estadísticamente, pero sí mostrar una tendencia.

Los estudios realizados han mostrado una prevalencia de signos y síntomas de TTM mayor en el sexo femenino ${ }^{(18,23,24)}$. Sin embargo, los datos obtenidos en este estudio no mostraron diferencias estadísticas según el sexo, aunque se observó al sexo femenino ligeramente más afectado al compararlo con el masculino. El signo más prevalente observado en ambos sexos fue la desviación mandibular, seguido por el ruido articular. Éste último fue encontrado en menor prevalencia que otros estudios ${ }^{(7,20)}$, esto podría deberse a la diferencia metodológica utilizada. El dolor fue el síntoma de menor prevalencia.

\section{Limitaciones del estudio}

Las limitaciones de la presente investigación estuvieron relacionadas con la metodología usada durante el examen físico en busca de signos y síntomas de TTM, debido a que no hubo una calibración previa entre los alumnos del postítulo de ortodoncia.

Se sugiere seguir investigando sobre TTM en pacientes con maloclusión utilizando un protocolo que sea ampliamente reconocido y confiable, como son los "Criterios diagnósticos de los trastornos temporomandibulares (CD/TTM) para aplicaciones clínicas y de investigación"(25), para poder aunar criterios y a futuro reunir información obtenida bajo una misma metodología, incluyendo adicionalmente exámenes imagenológicos para una mayor sensibilidad diagnóstica en las alteraciones de tipo articular.

\section{Implicancias clínicas de los hallazgos encontrados}

Debido a que los TTM son una condición tan común en pacientes con maloclusión, es necesaria la evaluación de la ATM tanto antes, durante y después a un tratamiento ortodóncico. Más aún, en un paciente portador de TTM, es de suma importancia, tanto del punto de vista técnico como legal, una evaluación con un especialista en disfunción previo al inicio del tratamiento de ortodoncia. De este modo poder establecer un adecuado plan de tratamiento que permita la corrección de ambos y minimizar el tiempo de intervención con el objetivo alcanzar resultados favorables en la estética facial, en la función articular y en los músculos masticatorios.

\section{CONCLUSIONES}

A partir de los resultados obtenidos en este estudio, se concluyó lo siguiente:

- La prevalencia de signos y síntomas de TTM de la muestra total de pacientes con maloclusión examinados en el Postítulo de Ortodoncia de la FOUCH, entre los años 2013 y 2015 , fue de un $43,9 \%$.

- El signo con mayor prevalencia fue la desviación mandibular, seguido por el ruido articular y el salto articular. El síntoma con menor prevalencia fue el dolor.

- Se determinó que la prevalencia de signos y síntomas de TTM se incrementa con la edad, encontrándose diferencia estadística entre los distintos grupos etarios $(p \leq 0,05)$.

- No se encontró diferencia estadística en la prevalencia de signos y/o síntomas de TTM por sexo $(p>0,05)$.

\section{CONFLICTO DE INTERESES}

Los autores declaran no tener ningún conflicto de interés en la realización del presente estudio 


\section{Bibliografía}

1. Romo F, Díaz W, Schulz R, Torres M. Tópicos de odontología integral [Internet] Santiago: Universidad de Chile; 2011. [citado: 2015, septiembre]. Disponible en: http://www.repositorio.uchile.cl/handle/2250/123378

2. Aliaga A, Mattos M, Aliaga R, Del Castillo C. Maloclusiones en niños y adolescentes de caseríos y comunidades nativas de la Amazonía de Ucayali, Perú. Rev Peru Med Exp. 2011;28:87-91.

3. Cartes R, Araya E, Valdés C. Maloclusiones y su impacto psicosocial en estudiantes de un liceo intercultural. Int J Odontostomat. 2010;4:65-70.

4. Sadowsky C, Theisen T, Sakols E. Orthodontic treatment and temporomandibular joint sounds -a longitudinal study. Am J Orthod Dentofacial Orthop. 1991;99:441-447. 5. Kurita H, Ohtsuta A, Kurashina K, Kopp S. A study of factors for successful splint capture of anteriorly disc displaced Temporomandibular joint disc with disc repositioning appliance. J Oral Rehab. 2001;28:651-657.

6. Morrant D, Taylor G. The prevalence of temporomandibular disorder in patients referred for the orthodontic assessment. Br J Orthod. 1996;23:261-5.

7. López V, Gómez G, Canseco J. Evaluación clínica de la disfunción temporomandibular antes del tratamiento ortodóncico. Rev Odont Mex. 2004:8:80-89. 8. Spalj S, Slaj M, Athanasiou A, Zak I, Simunovic M. Temporomandibular disorders and orthodontic treatment need in orthodontically untreated children and adolescents. Coll Antropol. 2015;39:151-8.

9. Soto L, De la Torre J, Aguirre I, De la Torre E. Trastornos temporomandibulares en pacientes con maloclusión. Facultad de Estomatología de la Universidad Médica de La Habana. La Habana, Cuba. Rev Cubana Estomatol. 2013; 50:374-387.

10. Richard C, Gantz R, Cabrera J, Ayala J. Las anomalías dentomaxilares. Daño poblacional y realidad social. Rev Dent Chile.1972;62:147-9.

11. Gazit E, Lieberman M, Eini R, Hirsch N, serfaty V, Fuchs C, Lilos P. Prevalence of mandibular dysfunction in 10-18 year old Israeli schoolchildren. J Oral Rehabil. 1984;11:307-317.

12. Pullinger A, Monteiro A. History factors associated with symptoms of temporomandibular disorders. J Oral Rehabil. 1988;15:117-24.

13. List T, Dworkin S. Comparing TMD diagnoses and clinical findings at Swedish and US TMD centers using research diagnostic criteria for temporomandibular disorders. J Orofac Pain. 1996;10:240-53.

14. Tallents R, Macher D, Kyrkanides S, Katzberg R, Moss M. Prevalence of missing posterior teeth and intraarticular temporomandibular disorders. J Prosthet Dent. 2002;87:45-50.
15. DeBont L, Digkraaf L, Stegenga B. Epidemiology and natural progression of articular temporomandibular disorders. J Oral Surg Oral Med Oral Pathol Ora Radiol and Endod. 1997;83:72-6.

16. Le Reche L. Epidemiology of temporomandibular disorders; Implication for the investigations of etiologic factors. Crit Rev Oral Biol Med. 1997;8:291-305.

17. Díaz W, Guzmán L, Ardila C. Prevalencia y necesidad de tratamiento de trastornos temporomandibulares en una población Chilena. AMC. 2012;16:602-609. 18. Casassus R. Prevalencia de trastornos temporomandibulares en escolares de 10 a 14 años de la provincia de Santiago, Región Metropolitana 2007. Tesis para optar al grado de Magíster en Ciencias Odontológicas con mención en Fisiología y Patología del Crecimiento y Desarrollo Maxilofacial. U. de Chile, Facultad de Odontología, Escuela de Graduados, 2007.

19. Corsini G, Fuentes R, Bustos L, Borie E, Navarrete A, Navarrete D, et al. Determinación de los signos y síntomas de los trastornos temporomandibulares, en estudiantes de 13 a 18 años de un colegio de la comuna de Temuco, Chile. Int $J$ Morphol. 2005;23:345-352.

20. Islas N, Villanueva N, Cuairán V, Canseco J. Disfunción de la articulación temporomandibular en pacientes de 9 a 14 años pretratamiento de ortodoncia. Rev Odont Mex. 2011;15:72-76.

21. Solberg W, Woo M, Houston J. Prevalence of mandibular dysfunction in young adults. J Am Dent Assoc. 1979:98:25-34.

22. Luther F. Orthodontics and the temporomandibular joint: Where are we now? Part 1. Orthodontic treatment and temporomandibular disorders. Angle Orthod.1988;68 295-304

23. Gerra G, Volpi R, Delsignore R. Sex -Related responses of beta endorphin ACTH, GH and PRL to cold exposure in humans. Acta Endocrinol. 1992;126:24-8. 24. Almagro I, Castro A, Matarán G, Quesada J, Quisado R, Moreno L. Temporomandibular joint dysfunction, disability and oral health in a communitydwellir elderly population. Nutr Hosp 2011;26:1045-1051.

25. Schiffman E, Ohrbach R, Truelove E, Look J, Anderson G, Goulet J, et al. Diagnostic Criteria for Temporomandibular Disorders (DC/TMD) for clinical and research applications: Recommendations of the International RCD/TMD Consortium Network and Orofacial Pain Special Interest Group. J Oral Facial Pain Headache. $2014 ; 28: 6-27$ 